\title{
La influencia de la religiosidad en la salud: el caso de los hábitos saludables/no saludables
}

\author{
The influence of religiosity on health: the case of \\ healthy/unhealthy habits
}

\section{A influência da religiosidade na saúde: o caso de hábitos saudáveis/insalubres}

\author{
Rocío De Diego Corderoํ, Manuel Guerrero Rodríguez ${ }^{2}$ \\ ${ }^{1}$ Doctora. Profesora en el Departamento de Enfermería. Facultad de Enfermería, Fisioterapia y Podología. \\ Universidad de Sevilla \\ ${ }^{2}$ Graduado en Enfermería por la Universidad de Sevilla. Enfermero en Residencia para personas Gravemente \\ Afectadas "Fundación Auxilia Sevilla" \\ Cómo citar este artículo en edición digital: De Diego Cordero, R. E Guerrero Rodríguez, M. (2018). La \\ influencia de la religiosidad en la salud: el caso de los hábitos saludables/no saludables. Cultura de los \\ Cuidados (Edición digital), 22(52). Recuperado de http://dx.doi.org/10.14198/cuid.2018.52.15 \\ Correspondencia: Departamento de Enfermería. Facultad de Enfermería, Fisioterapia y Podología. \\ Universidad de Sevilla. Cl Avenzoar, n. ${ }^{\circ} 61009$ Sevilla. España \\ Correo electrónico: rdediego2@us.es \\ Recibido: 02/04/2018; Aceptado: 10/06/2018
}

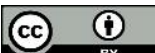

\section{ABSTRACT}

Introduction: In Spain, the religious pluralism can became a challenge to healthcare professionals in their role of providing coherent care and attention that respects the beliefs and values of usersen.

Objective: The objective of this study is to know the influence of religiosity / spirituality on behavior patterns in relation to lifestyles: healthy and unhealthy habits.

Methodology: Systematic literature review published between 2012-2017 and it searched in databases: SCOPUS, PUBMED, LILACS and WOS.

Results: The 22 selected articles showed a direct relationship between the level of religiosity and the follow-up of healthy behaviors in general, as well as the protective character of the life styles in the appearance of diseases. Also, a low consumption of toxic substances was observed in people with greater religious participation.

Conclusion: It can be affirmed that the affiliation to a religion does imply the realization of behavioral patterns that are usually beneficial to health. In addition, non-religious individuals with high levels of spirituality are also likely to engage in healthier behaviors than those with poorer levels.

Keywords: Religious, health behaviour, toxic habits, culture.

\section{RESUMO}

Introdução: O pluralismo religioso atual na Espanha torna-se um desafio para os profissionais de saúde em seu papel de 
oferecer atenção e cuidados coerentes que respeitem as crenças e os valores dos usuários.

Objetivo: O objetivo deste estudo é conhecer a influência da religiosidade/espiritualidade nos padrões de comportamento em relação aos estilos de vida: hábitos saudáveis e não saudáveis.

Metodologia: Revisão sistemática da literatura científica compreendida entre os años 2012-2017 nas bases de dados SCOPUS, PUBMED, LILACS e WOS.

Resultados: Os 22 artigos selecionados mostraram, em geral, uma relação direta entre o nível de religiosidade e o monitoramento de comportamentos saudáveis, bem como a natureza protetora dos estilos de vida em face do aparecimento de doenças. Do mesmo modo, observou-se um baixo consumo de substâncias tóxicas em pessoas com maior participação religiosa.

Conclusão: Pode-se afirmar que a afiliação a uma religião implica a realização de padrões de comportamento, principalmente benéficos para a saúde. Além disso, indivíduos não religiosos com altos níveis de espiritualidade também são susceptíveis de realizar comportamentos mais saudáveis do que aqueles com níveis mais deficientes.

Palavras chave: Religiosidade, comportamentos saudáveis, hábitos tóxicos, cultura.

\section{RESUMEN}

Introducción: El pluralismo religioso actual en España se convierte en un reto para los profesionales de la salud en su papel de ofrecer una atención y cuidados coherentes que respeten las creencias y valores de los usuarios.

Objetivo: El objetivo de este estudio conocer la influencia de la religiosidad/espiritualidad en las pautas de comportamiento en relación con los estilos de vida: hábitos saludables y no saludables.

Metodología: Revisión sistemática de la literatura científica comprendida entre los años 2012-2017 en las bases de datos SCOPUS, PUBMED, LILACS y WOS.

Resultados: Los 22 artículos seleccionados mostraron en general una relación directa entre el nivel de religiosidad y el seguimiento de conductas saludables, así como el carácter protector de los estilos de vida ante la aparición de enfermedades. Igualmente se observó un bajo consumo de sustancias tóxicas en personas con mayor participación religiosa.

Conclusión: Se puede afirmar que la filiación a una religión implica la realización de unos patrones de conducta, en su mayoría, beneficiosos para la salud. Además, individuos no religiosos pero con altos niveles de espiritualidad también son susceptibles de llevar a cabo conductas más saludables que aquellos con niveles más deficientes.

Palabras clave: Religiosidad, conductas saludables, hábitos tóxicos, cultura.

\section{INTRODUCCIÓN}

La salud debe ser entendida no sólo desde lo biológico, sino también desde su trascendencia social y cultural. El imperante modelo biomédico ha circunscrito la definición de salud a la ausencia de enfermedad, ignorando las esferas sociales y culturales que influyen de manera directa y que la antropología médica se ha preocupado por señalar (Moreno, 2008). La concepción de los términos salud, enfermedad y las acciones que los rodean (particular de cada cultura y momento 
social) puede denominarse como proceso de salud-enfermedad-atención. (Menéndez, 1994). En este proceso la religiosidad es un factor determinante dado que va a influir en cada uno de sus componentes, entendiendo a la misma como un sistema de creencias, prácticas, rituales y símbolos que facilita la cercanía con lo transcendente (Dios, poder divino o última verdad); se hace necesaria la distinción respecto al concepto de espiritualidad, que hace referencia a la búsqueda propia para responder a preguntas del significado de la vida, y que puede conducir o no a desarrollar creencias religiosas y formar parte una comunidad (Navas y Villegas, 2006).

Así, existen determinadas conductas (relacionadas con el consumo de tabaco, de alcohol, la higiene, el descanso o el ayuno) que de alguna forma siempre han tratado de ser mediadas por las diferentes religiones. La mayoría de las religiones suele creer en la dualidad cuerpo-alma. Desde esta perspectiva, la espiritualidad o la religiosidad sería todo aquello relacionado con el alma, el componente más importante y sagrado que se supone existe en el ser humano. El cuerpo, por su parte, suele asociarse con lo terrenal. Sin embargo, el cuerpo también puede estimarse como importante, incluso sagrado, ya que el cuidado del cuerpo favorecerá la vida espiritual. Por ello, las diferentes religiones suelen aconsejar la práctica de comportamientos específicos que favorecen la salud del cuerpo, como pueden ser la toma de algunos alimentos y la abstinencia de otros, o la prohibición de determinadas bebidas u otras sustancias (Planet, 2012).

De este modo, el Islam prohíbe el consumo, entre otros, de cerdo, jabalí (alimentos muy grasos), café, té y bebidas alcohólicas. En cuanto a la práctica de medidas higiénicas destaca la circuncisión de los varones, la cual consideran un método para prevenir infecciones. Los judíos también comparten la circuncisión $\mathrm{y}$ respecto a la dieta destaca el modo de preparación (rito judío) y conservación (proceso de salado) (Carrasco, 2015). Los miembros de la Iglesia de Jesucristo de los Santos de los Últimos días (conocidos como mormones) deben consumir alimentos nutritivos como frutas $y$ verduras saludables, limitar el consumo de carne roja, rechazar las drogas lícitas e ilícitas y descansar el tiempo necesario para fortificar el cuerpo y la mente (De Diego y Badanta, 2017). El cristianismo ortodoxo se caracteriza por la cantidad de ayuno que realizan, presente en más de la mitad del año (Koufakis, 2017). Ante la situación de pluralismo religioso actual, los profesionales de la salud deben reconocer la presencia de las diferencias culturales marcadas en este caso por la religiosidad en la población para ofrecer una atención coherente con las necesidades de salud de los usuarios dentro de su contexto, respetando y valorando dicha diversidad cultural (Da Trinidade et al.., 2013). Por ello, se plantea como objetivo de este estudio conocer la influencia de la religiosidad/espiritualidad en las pautas de comportamiento en relación con los estilos de vida: hábitos saludables y no saludables.

\section{METODOLOGÍA}

Se llevó a cabo un estudio exploratorio realizado a través de una revisión de la literatura científica siguiendo las recomendaciones de la Declaración PRISMA en las bases de datos SCOPUS, WOS, PUBMED y LILACS. La estrategia de búsqueda fue elaborada con los descriptores "religion", "health behaviour", "toxic habits", combinados mediante los booleanos "AND" y "OR" (Tabla 1). 
TABLA 1: Estrategias de búsqueda utilizadas

\begin{tabular}{|c|c|c|c|}
\hline Base de Datos & Estrategia & $\begin{array}{l}\text { Artículos } \\
\text { encontrados }\end{array}$ & $\begin{array}{l}\text { Artículos } \\
\text { incluidos }\end{array}$ \\
\hline PUBMED & $\begin{array}{c}\text { [religion OR religious] AND ("health } \\
\text { behaviour" OR "toxic habits) }\end{array}$ & 134 & 3 \\
\hline SCOPUS & $\begin{array}{l}\text { religion AND ("health behaviour" OR } \\
\text { "toxic habits ) }\end{array}$ & 1535 & 11 \\
\hline LILACS & Religio* AND salud* & 1032 & 7 \\
\hline WOS & $\begin{array}{l}\text { Religio* AND ["health behavior" OR } \\
\text { "toxic habits"] }\end{array}$ & 93 & 4 \\
\hline
\end{tabular}

FUENTE: Elaboración propia

\section{RESULTADOS}

Se han analizado 22 artículos (2 revisiones sistemáticas, 12 estudios descriptivos transversales, 2 estudios correlacionales, 2 estudios etnográficos, 2 estudios de regresión logística, 1 estudio prospectivo y 1 estudio de cohortes) (Tabla 2). Los principales hallazgos identificados durante el análisis fueron "la relación entre estilos de vida saludables y la aparición de patologías" (A); "la distribución del consumo de drogas lícitas e ilícitas en adeptos de alguna religión frente a los no adeptos" (B); y "la creencia de una religión como condición que favorece el mayor o menor seguimiento de conductas de salud" (C).

TABLA 2: Resumen de los estudios seleccionados

\begin{tabular}{|c|c|c|c|c|c|}
\hline Autor* & Año/País & Metodología & Objetivos & $\begin{array}{l}\text { Principales } \\
\text { Resultados }\end{array}$ & $\begin{array}{c}\text { Principales } \\
\text { hallazgos }\end{array}$ \\
\hline $\begin{array}{l}\text { Billioux VG, } \\
\text { Sherman SG } \\
\text { et al. }\end{array}$ & $\begin{array}{l}\text { 2012/Estados } \\
\text { Unidos }\end{array}$ & $\begin{array}{l}\text { Análisis de } \\
\text { regresión } \\
\text { logística } \\
\text { univariante } \\
\text { multivariante }\end{array}$ & $\begin{array}{l}\text { Examinar si la } \\
\text { religiosidad- } \\
\text { ideación religiosa, } \\
\text { participación } \\
\text { religiosa y el apoyo } \\
\text { religioso están } \\
\text { asociados con los } \\
\text { hábitos tóxicos. }\end{array}$ & $\begin{array}{lr}\text { La religiosidad tiene } \\
\text { una asociación inversa } \\
\text { con el consumo de } \\
\text { drogas } \\
\text { comportamientos de } \\
\text { riesgo entre las } \\
\text { comunidades con altas } \\
\text { tasas de consumo de } \\
\text { drogas. }\end{array}$ & B \\
\hline $\begin{array}{l}\text { Brewer G., } \\
\text { Robinson S. } \\
\text { et al. }\end{array}$ & $\begin{array}{l}\text { 2015/Reino } \\
\text { Unido }\end{array}$ & $\begin{array}{l}\text { Estudio } \\
\text { descriptivo } \\
\text { transversal }\end{array}$ & $\begin{array}{l}\text { Investigar la } \\
\text { influencia del apoyo } \\
\text { social religioso en } \\
\text { una serie de } \\
\text { comportamientos, } \\
\text { estados y actitudes } \\
\text { de salud en mujeres } \\
\text { y hombres } \\
\text { cristianos. }\end{array}$ & $\begin{array}{l}\text { El apoyo religioso no } \\
\text { influyó en la actividad } \\
\text { física pero sí mejoraba } \\
\text { la resistencia a la } \\
\text { enfermedad. } \\
\text { afrontamiento } \\
\text { religioso negativo era } \\
\text { predictivo de un } \\
\text { aumento del consumo } \\
\text { de alcohol. }\end{array}$ & $\mathrm{C}$ \\
\hline $\begin{array}{l}\text { Bruce MA } \\
\text { et al. }\end{array}$ & $\begin{array}{l}\text { 2016/Estados } \\
\text { Unidos }\end{array}$ & $\begin{array}{l}\text { Estudio } \\
\text { prospectivo. }\end{array}$ & $\begin{array}{l}\text { Examinar la relación } \\
\text { entre } \\
\text { religiosidad/espiritu } \\
\text { alidad y los } \\
\text { comportamientos } \\
\text { relacionados con la } \\
\text { obesidad en varones } \\
\text { adolescentes } \\
\text { afroamericanos }\end{array}$ & $\begin{array}{l}\text { La espiritualidad actúa } \\
\text { como un factor } \\
\text { protector más potente } \\
\text { que la religiosidad para } \\
\text { reducir el riego de } \\
\text { obesidad. }\end{array}$ & A \\
\hline
\end{tabular}




\begin{tabular}{|c|c|c|c|c|c|}
\hline Autor* & Año/País & Metodología & Objetivos & $\begin{array}{l}\text { Principales } \\
\text { Resultados }\end{array}$ & $\begin{array}{c}\text { Principales } \\
\text { hallazgos }\end{array}$ \\
\hline $\begin{array}{l}\text { Caravaca } \\
\text { Morera JA } \\
\text { et al. }\end{array}$ & 2015/Canadá & $\begin{array}{l}\text { Estudio } \\
\text { multicéntrico } \\
\text { transversal }\end{array}$ & $\begin{array}{l}\text { Explorar la relación } \\
\text { entre factores } \\
\text { culturales (religión, } \\
\text { espiritualidad, } \\
\text { relaciones familiares } \\
\text { y con compañeros) y } \\
\text { el consumo de } \\
\text { drogas en en } \\
\text { universitarios de } 5 \\
\text { países } \\
\text { latinoamericanos }\end{array}$ & $\begin{array}{l}\text { El nivel de } \\
\text { espiritualidad influye } \\
\text { de forma positiva en un } \\
\text { menor Aconsumo de } \\
\text { drogas lícitas (alcohol } \\
\text { y tabaco), pero no } \\
\text { mostró significancia en } \\
\text { el consumo de drogas } \\
\text { ilícitas }\end{array}$ & 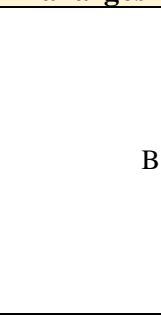 \\
\hline $\begin{array}{l}\text { Castellanos } \\
\text { Soriano F., } \\
\text { Lucero López } \\
\text { A. }\end{array}$ & $\begin{array}{l}\text { 2012/Colomb } \\
\text { ia }\end{array}$ & $\begin{array}{l}\text { Estudio } \\
\text { cualitativo }\end{array}$ & $\begin{array}{l}\text { Presentar hallazgos } \\
\text { en torno a las } \\
\text { prácticas religiosas } \\
\text { católicas en un grupo } \\
\text { de personas mayores } \\
\text { en situación de } \\
\text { discapacidad y } \\
\text { pobreza y su impacto } \\
\text { en el cuidado de la } \\
\text { salud. }\end{array}$ & $\begin{array}{l}\text { La religión ejerce en } \\
\text { las personas mayores } \\
\text { una influencia positiva, } \\
\text { provee fortaleza y } \\
\text { valor; y proporciona } \\
\text { elementos para que } \\
\text { afronten su situación }\end{array}$ & $\mathrm{C}$ \\
\hline $\begin{array}{l}\text { Da Rocha } \\
\text { Quieroz N. } \\
\text { et al. }\end{array}$ & 2015/Brasil & $\begin{array}{l}\text { Estudio } \\
\text { transversal }\end{array}$ & $\begin{array}{l}\text { Verificar la } \\
\text { asociación entre la } \\
\text { religiosidad y el } \\
\text { patrón de consumo } \\
\text { de alcohol y tabaco } \\
\text { en lo usuarios de los } \\
\text { servicios de atención } \\
\text { primaria }\end{array}$ & $\begin{array}{l}\text { Se observó una } \\
\text { asociación entre la } \\
\text { religiosidad intrínseca } \\
\text { como factor protector } \\
\text { en relación con el } \\
\text { consumo moderado y } \\
\text { alto de alcohol y tabaco }\end{array}$ & B \\
\hline $\begin{array}{l}\text { Debnam K. } \\
\text { et al. }\end{array}$ & $\begin{array}{l}\text { 2012/Estados } \\
\text { Unidos }\end{array}$ & $\begin{array}{l}\text { Estudio } \\
\text { cualitativo }\end{array}$ & $\begin{array}{lr}\text { Examinar } & \text { las } \\
\text { contribuciones } & \text { del } \\
\text { apoyo social } & \text { y } \\
\text { religioso a } & \text { una } \\
\text { variedad } & \text { de } \\
\text { comportamientos de } \\
\text { salud. }\end{array}$ & $\begin{array}{lr}\text { El apoyo religioso } \\
\text { predijo un mayor } \\
\text { consumo de frutas y } \\
\text { verduras, realización } \\
\text { de actividad física } \\
\text { moderada } \\
\text { disminución y } \\
\text { consumo de alcohol. }\end{array}$ & $\mathrm{C}$ \\
\hline $\begin{array}{l}\text { Gabriela Rusa } \\
\text { S. } \\
\text { et al. }\end{array}$ & 2014/Brasil & $\begin{array}{l}\text { Estudio } \\
\text { descriptivo, de } \\
\text { corte transversal } \\
\text { y con abordaje } \\
\text { cuantitativo }\end{array}$ & $\begin{array}{lr}\text { Evaluar la calidad de } \\
\text { vida/ espiritualidad, } \\
\text { religión y creencias } \\
\text { personales } & \text { de } \\
\text { pacientes renales } \\
\text { crónicos r } \\
\text { hemodiálisis }\end{array}$ & $\begin{array}{l}\text { La espiritualidad, la } \\
\text { religión y las creencias } \\
\text { personales actúan } \\
\text { como herramientas } \\
\text { importantes en el } \\
\text { afrontamiento de la } \\
\text { enfermedad renal } \\
\text { crónica y diálisis. }\end{array}$ & A \\
\hline $\begin{array}{l}\text { Garcia G., } \\
\text { Ellison CG } \\
\text { et al. }\end{array}$ & $\begin{array}{l}\text { 2012/Estados } \\
\text { Unidos }\end{array}$ & $\begin{array}{l}\text { Estudio } \quad \text { de } \\
\text { regresión } \\
\text { logística }\end{array}$ & $\begin{array}{l}\text { Comprobar la } \\
\text { influencia protectora } \\
\text { que ofrece la religión } \\
\text { respecto a ciertos } \\
\text { resultados de salud } \\
\text { (consumo de alcohol } \\
\text { y tabaco) en una } \\
\text { población de Texas. }\end{array}$ & $\begin{array}{l}\text { Protestantes con gran } \\
\text { participación religiosa } \\
\text { son más propensos a } \\
\text { ser abstemios y no } \\
\text { fumadores. Aquellos } \\
\text { sin afiliación religiosa } \\
\text { exhiben perfiles de } \\
\text { riesgo relativamente } \\
\text { desfavorables. }\end{array}$ & B \\
\hline Glicksman G. & $\begin{array}{l}\text { 2017/Estados } \\
\text { Unidos. }\end{array}$ & $\begin{array}{l}\text { Estudio } \\
\text { descriptivo } \\
\text { transversal }\end{array}$ & $\begin{array}{l}\text { Determinar si el } \\
\text { estado } \\
\text { comportamientos de } \\
\text { salud de judíos } \\
\text { mayores difieren de } \\
\text { los no judíos. }\end{array}$ & $\begin{array}{l}\text { Los judíos presentan } \\
\text { mejores } \\
\text { comportamientos de } \\
\text { salud que católicos y } \\
\text { protestantes, a pesar de } \\
\text { asistir menos a los } \\
\text { servicios religiosos que } \\
\text { las otras dos } \\
\text { tradiciones. }\end{array}$ & $\mathrm{C}$ \\
\hline $\begin{array}{l}\text { Harold G. } \\
\text { et al. }\end{array}$ & $\begin{array}{l}\text { 2015/Estados } \\
\text { Unidos }\end{array}$ & $\begin{array}{l}\text { Revisión } \\
\text { bibliográfica }\end{array}$ & $\begin{array}{l}\text { Conocer las últimas } \\
\text { investigaciones } \\
\text { sobre religión, } \\
\text { espiritualidad y } \\
\text { conductas de salud. }\end{array}$ & $\begin{array}{l}\text { Se encontraron } \\
\text { asociaciones inversas } \\
\text { entre } \\
\text { religiosidad/espiritualid } \\
\text { ad y consumo de tabaco, } \\
\text { alcohol, drogas ilícitas y } \\
\text { consumo excesivo de } \\
\text { drogas con receta. }\end{array}$ & B \\
\hline
\end{tabular}




\begin{tabular}{|c|c|c|c|c|c|}
\hline Autor* & Año/País & Metodología & Objetivos & $\begin{array}{l}\text { Principales } \\
\text { Resultados }\end{array}$ & $\begin{array}{l}\text { Principales } \\
\text { hallazgos }\end{array}$ \\
\hline $\begin{array}{l}\text { Hasnah } \\
\text { Hassan S. }\end{array}$ & 2015/Malasia & $\begin{array}{l}\text { Estudio } \\
\text { etnográfico }\end{array}$ & $\begin{array}{l}\text { Examinar las } \\
\text { relaciones entre los } \\
\text { aspectos religiosos y } \\
\text { los estilos de vida } \\
\text { relacionados con la } \\
\text { salud de los } \\
\text { musulmanes de } \\
\text { Malasia. }\end{array}$ & $\begin{array}{l}\text { El islam pide a sus } \\
\text { seguidores que tengan } \\
\text { una buena condición } \\
\text { física, dieta sana y la } \\
\text { restricción de ciertos } \\
\text { alimentos, así como la } \\
\text { oración grupal que } \\
\text { favorece las relaciones } \\
\text { sociales. }\end{array}$ & $\mathrm{C}$ \\
\hline $\begin{array}{l}\text { Hayward RD } \\
\text { et al. }\end{array}$ & $\begin{array}{l}\text { 2017/Estados } \\
\text { Unidos }\end{array}$ & $\begin{array}{l}\text { Estudio } \\
\text { cualitativo }\end{array}$ & $\begin{array}{l}\text { Examinar la } \\
\text { prevalencia de dos } \\
\text { creencias religiosas: } \\
\text { la creencia en } \\
\text { milagros de curación } \\
\text { y el retraso de la } \\
\text { responsabilidad de } \\
\text { los resultados de } \\
\text { salud a Dios. }\end{array}$ & $\begin{array}{l}\text { Estas creencias pueden } \\
\text { tener efectos negativos } \\
\text { y positivos respecto a } \\
\text { los comportamientos } \\
\text { de salud. Destaca el } \\
\text { papel modulador de los } \\
\text { líderes religiosos. }\end{array}$ & $\mathrm{C}$ \\
\hline $\begin{array}{l}\text { Janssen- } \\
\text { Niemeijer JA } \\
\text { et al. }\end{array}$ & $\begin{array}{l}\text { 2017/Países } \\
\text { Bajos }\end{array}$ & $\begin{array}{l}\text { Revisión } \\
\text { bibliográfica de } \\
\text { estudios } \\
\text { cualitativos }\end{array}$ & $\begin{array}{l}\text { Obtener una } \\
\text { comprensión de lo } \\
\text { que se conoce sobre } \\
\text { el papel de } \\
\text { espiritualidad en los } \\
\text { cambios de estilo de } \\
\text { vida del paciente con } \\
\text { enfermedad } \\
\text { cardiovascular }\end{array}$ & $\begin{array}{l}\text { La espiritualidad es } \\
\text { importante cuando se } \\
\text { requieren cambios de } \\
\text { estilo de vida en el } \\
\text { debut de enfermedades } \\
\text { cardiovasculares. } \\
\text { Puede dar apoyo, pero } \\
\text { también angustia, } \\
\text { culpabilidad... }\end{array}$ & A \\
\hline $\begin{array}{l}\text { Kretchy I., } \\
\text { Owusu- } \\
\text { Daaku F. } \\
\text { et al. }\end{array}$ & 2013/Ghana & $\begin{array}{l}\text { Estudio } \\
\text { descriptivo } \\
\text { transversal }\end{array}$ & \begin{tabular}{lrr} 
Comprobar & si & altas \\
puntuaciones & de \\
creencias & \\
espirituales & \\
prácticas & religiosas \\
influyen & en & la \\
decisión & de & los \\
pacientes & & de \\
adherirse & & al \\
tratamiento & médico \\
\multicolumn{2}{l}{ hipertensivo. }
\end{tabular} & 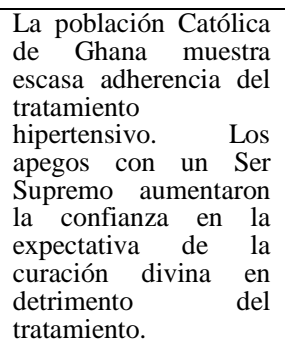 & $\mathrm{C}$ \\
\hline $\begin{array}{l}\text { Linardakis } \\
\text { M., } \\
\text { Papadaki A. } \\
\text { et al. }\end{array}$ & 2015/Grecia & $\begin{array}{l}\text { Estudio } \\
\text { transversal }\end{array}$ & $\begin{array}{l}\text { Examinar la relación } \\
\text { entre las creencias } \\
\text { religiosas/espirituale } \\
\text { s y la adopción y } \\
\text { presencia de factores } \\
\text { de riesgo para } \\
\text { enfermedades } \\
\text { crónicas en adultos } \\
\text { europeos. }\end{array}$ & $\begin{array}{lrr}\text { Las personas con } \\
\text { mayor religiosidad } \\
\text { poseen estilos de vida } \\
\text { más saludables } \\
\text { menos factores de } \\
\text { riesgo para el } \\
\text { desarrollo } & \text { de } \\
\text { enfermedades crónicas }\end{array}$ & A \\
\hline $\begin{array}{l}\text { Lopes Chaves } \\
\text { EC } \\
\text { et al. }\end{array}$ & 2014/Brasil & $\begin{array}{l}\text { Estudio } \\
\text { descriptivo } \\
\text { transversal }\end{array}$ & $\begin{array}{lr}\text { Investigar la calidad } \\
\text { de vida y síntomas } \\
\text { depresivos } & \text { en } \\
\text { ancianos y } & \text { su } \\
\text { relación con } & \text { la } \\
\text { religiosidad } & \\
\end{array}$ & $\begin{array}{l}\text { A mayor participación } \\
\text { religiosa, mayor } \\
\text { calidad de vida, pero } \\
\text { sin relación con la } \\
\text { presencia de síntomas } \\
\text { depresivos. }\end{array}$ & A \\
\hline $\begin{array}{l}\text { Padela AI } \\
\text { et al. }\end{array}$ & $\begin{array}{l}\text { 2016/Estados } \\
\text { Unidos. }\end{array}$ & $\begin{array}{l}\text { Estudio } \\
\text { cualitativo }\end{array}$ & $\begin{array}{lr}\text { Identificar } & \text { la } \\
\text { relación entre las } \\
\text { creencias religiosas } \\
\text { y la realización de } \\
\text { mamografía } & \text { en } \\
\text { musulmanas } & \text { de } \\
\text { Chicago. } & \end{array}$ & $\begin{array}{l}\text { Las creencias islámicas } \\
\text { influyen tanto positiva } \\
\text { como negativamente en } \\
\text { el cribado del cáncer. } \\
\text { Los mensajes de los } \\
\text { líderes religiosos pueden } \\
\text { mejorar la detección del } \\
\text { cáncer de mama. }\end{array}$ & $\mathrm{C}$ \\
\hline
\end{tabular}




\begin{tabular}{|c|c|c|c|c|c|}
\hline Autor* & Año/País & Metodología & Objetivos & $\begin{array}{l}\text { Principales } \\
\text { Resultados }\end{array}$ & $\begin{array}{c}\text { Principales } \\
\text { hallazgos }\end{array}$ \\
\hline $\begin{array}{l}\text { Pitel L., } \\
\text { Maradasova } \\
\text { Geckova A. } \\
\text { et al. }\end{array}$ & $\begin{array}{l}\text { 2012/Eslovaq } \\
\text { uia }\end{array}$ & $\begin{array}{l}\text { Estudio } \\
\text { analítico } \\
\text { descriptivo }\end{array}$ & $\begin{array}{lr}\begin{array}{l}\text { Evaluar la relación } \\
\text { entre }\end{array} & \text { el } \\
\text { comportamiento } \\
\text { relacionado con la } \\
\text { salud y } \\
\text { religiosidad la } \\
\text { adolescentes } \\
\text { eslovacos, } \\
\text { evaluando el grado } \\
\text { en el que el género } \\
\text { afecta a esta } \\
\text { asociación. }\end{array}$ & $\begin{array}{l}\text { Existe relación inversa } \\
\text { entre religiosidad y } \\
\text { hábito no saludables. } \\
\text { La asociación es más } \\
\text { significativa en niñas } \\
\text { en el consumo de } \\
\text { tabaco, alcohol, } \\
\text { cannabis y absentismo } \\
\text { escolar. }\end{array}$ & B \\
\hline $\begin{array}{l}\text { Wang Z., } \\
\text { Koenig HG } \\
\text { et al. }\end{array}$ & 2015/China & $\begin{array}{l}\text { Estudio } \\
\text { correlacional }\end{array}$ & $\begin{array}{l}\text { Examinar la } \\
\text { asociación entre la } \\
\text { participación } \\
\text { religiosa y el } \\
\text { consumo de tabaco } \\
\text { en una comunidad } \\
\text { musulmana de China } \\
\text { y comparación con la } \\
\text { población no } \\
\text { musulmana. }\end{array}$ & $\begin{array}{l}\text { La participación } \\
\text { religiosa está } \\
\text { inversamente con el } \\
\text { relacionada con } \\
\text { tabaquismo, sobre todo } \\
\text { en varones } \\
\text { musulmanes que } \\
\text { asisten a actividades } \\
\text { religiosas más de una } \\
\text { vez por semana. }\end{array}$ & B \\
\hline $\begin{array}{l}\text { Watkins YJ, } \\
\text { Quinn LT } \\
\text { et al. }\end{array}$ & $\begin{array}{l}\text { 2013/ Estados } \\
\text { Unidos }\end{array}$ & $\begin{array}{ll}\text { Estudio } & \text { de } \\
\text { cohorte } & \text { de } \\
\text { diseño } & \\
\text { transversal } & \end{array}$ & $\begin{array}{l}\text { Investigar la relación } \\
\text { entre creencias y } \\
\text { prácticas espirituales } \\
\text { y religiosas, apoyo } \\
\text { social y actividades } \\
\text { de autocuidado de la } \\
\text { diabetes en } \\
\text { afroamericanos con } \\
\text { DMT2 }\end{array}$ & $\begin{array}{l}\text { Se encontraron } \\
\text { relaciones } \\
\text { significativas en } \\
\text { cuanto la dieta y el } \\
\text { cuidado de los pies. No } \\
\text { se encontró relación } \\
\text { con la realización de } \\
\text { actividad física. }\end{array}$ & $\mathrm{C}$ \\
\hline $\begin{array}{l}\text { Zini A., } \\
\text { Sgan-Cohen } \\
\mathrm{HD} \\
\text { et al. }\end{array}$ & 2012/Israel & $\begin{array}{l}\text { Estudio } \\
\text { descriptivo } \\
\text { transversal }\end{array}$ & $\begin{array}{l}\text { Evaluar la relación } \\
\text { entre la religiosidad } \\
\text { y la salud oral. }\end{array}$ & $\begin{array}{l}\text { En la población judía } \\
\text { existe una relación } \\
\text { significativa entre la } \\
\text { religiosidad y la } \\
\text { higiene a bucal, } \\
\text { posiblemente debida a } \\
\text { una buena dieta (sin } \\
\text { excesos de azúcar) y la } \\
\text { ausencia } \\
\text { tabaquismo. }\end{array}$ & $\mathrm{C}$ \\
\hline
\end{tabular}

FUENTE: Elaboración propia

\section{DISCUSIÓN}

\section{Relación entre estilos de vida saludables y la aparición de patologías}

Se pueden destacar artículos (Linardakis et al., 2015), (Bruce et al., 2016) que confluyen en que los estilos de vida de los sujetos estudiados actúan como un factor protector ante determinadas enfermedades. Por otro lado, existen aportaciones (Lopes et al., 2014) que coinciden con los autores anteriores en que cuanto mayor es la participación religiosa del individuo, mayor calidad de vida presenta. Sin embargo discrepan en la relación de este factor con la aparición de trastornos mentales. Dicha participación religiosa es entendida como las actitudes religiosas de las personas creyentes, las cuales pueden ser evaluadas mediante escalas que miden la asistencia al culto, frecuencia de las oraciones, participación en rituales, etc. Destacan "Escala de Actitud Religiosa" o "The Duke University Religion Index". (Orozco-Parra, 2014).

En fases más avanzadas del proceso de salud-enfermedad, la religiosidad no solo previene la aparición de la problemas, sino que facilita a la persona con manifestaciones patológicas ya presentes el cambio en los estilos de vida para prevenir la aparición de complicaciones (Janssen-Niemeijer et al., 2017) y le proporciona una mayor capacidad de afrontamiento, pues aporta al individuo 
confianza en sí mismo y fuerza de voluntad (Gabriela et al., 2015).

\section{Distribución del consumo de drogas lícitas e ilícitas en adeptos de alguna religión frente a los no adeptos}

Tras analizar los artículos se puede afirmar que los individuos más religiosos y/o con mayor espiritualidad tienen menos riesgo de consumir estas sustancias que aquellos que se consideran ateos, agnósticos o que presentan menor nivel de espiritualidad. De este modo, cabe señalar aportaciones (Da Rocha et al., 2015), (García et al., 2013) que establecen la existencia de una relación inversa entre la participación religiosa y el consumo de alcohol y tabaco. Otro estudio incorpora al análisis el consumo de cannabis y el absentismo escolar, que bajo una perspectiva de género concluye que la relación inversa entre religiosidad y hábitos no saludables es más significativa en chicas que en chicos (Pitel et al., 2012).

Billioux et al. (2014) centran su investigación en el consumo de drogas y la adopción de comportamientos de riesgo, como puede ser contraer VIH por la compartición de jeringuillas, obteniendo resultados similares que los autores citados en el párrafo anterior (inversa la relación entre la religiosidad y el consumo de drogas y/o comportamientos de riesgo). Siguiendo la misma línea, un estudio que analiza la relación entre el tabaquismo y el Islam, revela que al comparar la población musulmana con la que no lo es se corroboran los estudios anteriormente mencionados: la población religiosa muestra menor consumo de tabaco que la no religiosa. Dicho resultado ha sido más significativo en varones que asisten más de una vez a la semana a actividades religiosas (Wang et al., 2015).

Otros estudios (Caravaca et al. 2015), (Koenig, 2015) comparten con los artículos anteriores la investigación sobre el consumo de alcohol, tabaco, drogas y comportamientos de riesgo. Sin embargo no se centran únicamente en la religiosidad en sí, como se ha podido comprobar hasta ahora. El primero muestra una asociación inversa entre los hábitos tóxicos y la espiritualidad, la cual fue significativa para conductas como consumo de alcohol y tabaco, pero no llego a ser significativa en el consumo de drogas ilícitas. En el caso del segundo autor, se abre un debate entre religiosidad y espiritualidad, concluyendo en que la religiosidad es un constructo más fácil de medir que la espiritualidad, para la cual se usan escalas que terminan valorando la participación religiosa.

\section{Creencia en una religión como condición que favorece el mayor o menor seguimiento de conductas de salud}

La mayor parte de la bibliografía consultada relaciona la filiación de una religión con el seguimiento de conductas saludables. De este modo, se ha observado una asociación positiva entre el judaísmo y la higiene oral, la cual es posiblemente debida al bajo consumo de alimentos excesivamente azucarados y a la restricción tabáquica (Zini et al., 2012). Otro estudio añade que los judíos presentan mejores comportamientos de salud que, por ejemplo, católicos y protestantes (más visitas al médico, más control de la tensión arterial, más pruebas de cribado de cáncer, etc.) (Glicksman, 2017). Sin embargo, a pesar de realizar mejores comportamientos presentan un menor índice de participación religiosa (dato que contradice a todos los artículos anteriores). Este hecho es explicado en base a que los judíos más ancianos identificaban a sus semejantes como un grupo cultural o un pueblo, más que una 
comunidad religiosa (Glicksman, 2017).

En el caso del cristianismo protestante, Watkins et al. (2013) exponen que una mayor religiosidad en diabéticos tipo II era sinónimo de una dieta adecuada y un buen cuidado de los pies. En cambio, esta asociación no fue significativa para la realización de actividad física (Brewer et al., 2014). Ambos autores niegan una relación positiva con la realización de actividad física. En el ámbito católico, Castellanos (2012) estudia en un grupo de personas mayores que la religión ejerce una influencia positiva en el autocuidado de la salud, pues les ofrece fortaleza y confianza para llevar a cabo conductas saludables (salir a pasear, evitar alimentos contraindicados por patologías de base, etc.).

En el caso del Islam, esta religión exige a sus fieles estrictos comportamientos saludables. Tener buena condición física, seguir una dieta sana restringiendo ciertos alimentos, así como la oración grupal que favorece las relaciones sociales son ejemplos de dichos comportamientos (Hassan, 2015). No obstante, la disposición de las mujeres a realizarse mamografías bienales no es tan positiva (Padela et al., 2016). Este autor señala que las ideas de curación divina, el destino en las manos de Dios y las preferencias por un proveedor de cuidados del mismo sexo condicionan la elección de las musulmanas para realizarse una mamografía. Asimismo, destaca la importancia de los mensajes de los líderes religiosos para promocionar este hábito.

En relación con lo anterior, Hayward (2017) también analiza la relación existente entre las ideas de curación divina y las conductas de salud, en su caso en un grupo de mayoría protestante. Los resultados obtenidos coinciden con Padela en que el desplazamiento de la responsabilidad de la salud a Dios puede tener efectos positivos y negativos, y por tanto la influencia de los líderes religiosos es fundamental para llevar a cabo conductas saludables. Kretchy (2013) obtiene similares resultados que los dos autores anteriores. Su estudio relaciona la no adherencia al tratamiento médico antihipertensivo de la población de Ghana (mayoritariamente Católica), con la confianza en un Ser Supremo y las expectativas de curación divina

Por último, Debnam (2012) centra su investigación en el apoyo religioso, el cual resulta más eficaz que el apoyo social en cuanto al seguimiento de conductas de salud, como el mayor consumo de frutas y verduras, la no ingesta de alcohol y la realización de ejercicio físico.

\section{CONCLUSIONES}

Tras analizar detalladamente todos los artículos seleccionados y dando respuesta a la pregunta de revisión se han obtenido las siguientes conclusiones:

1) Los estilos de vida que proponen las distintas religiones estudiadas suponen un factor protector contra la aparición de enfermedades y problemas de salud. Una mayor participación religiosa, conlleva a darle gran importancia a las pautas de comportamiento recomendadas por cada religión, es decir, a tener unos estilos de vidas saludables: llevar una dieta sana, hacer ejercicio físico, no consumir sustancias tóxicas, etc. No obstante, estas pautas no se limitan a factores físicos únicamente. La capacidad de afrontamiento, la esperanza y la resiliencia son conceptos que favorecen y facilitan el proceso salud-enfermedad-atención de las personas. 
2) Existe una relación inversa entre el nivel de religiosidad/espiritualidad y el consumo de sustancias tóxicas (alcohol, tabaco, drogas ilegales, comportamientos de riesgo). Incluso individuos que no se identifican con ninguna filiación religiosa, pero con ciertos niveles de espiritualidad, son menos susceptibles a tener estos hábitos tóxicos.

3) Es importante recalcar la influencia que adquieren los líderes religiosos en la toma decisiones relacionadas con la propia salud, sobre todo en cuestiones de tratamientos o pruebas diagnósticas, muchas veces eclipsadas por creencias de curación divina o de desplazamiento de la responsabilidad de la propia salud a un Ser Supremo.

Respondiendo a la pregunta de revisión, se puede afirmar que la filiación a una religión sí implica la realización de unos patrones de conducta, en su inmensa mayoría, beneficiosos para la salud. Además, individuos no religiosos pero con altos niveles de espiritualidad también son candidatos a llevar a cabo conductas más saludables que aquellos con niveles más deficientes.

Somos conscientes de los numerosos debates a desarrollar en relación al tema planteado como la eficacia del apoyo social y el apoyo religioso en relación al seguimiento de conductas de salud en el que ajustándonos a la extensión requerida para este artículo, no hemos podido profundizar pero que sin duda supone una oportunidad para futuras investigaciones.

\section{BIBLIOGRAFÍA}

- Billioux, V.G., Sherman, S.G. \& Latkin, C. (2014). Religiosity and HIV-Related Drug Risk Behavior: A Multidimensional Assessment of Individuals from Communities with High Rates of Drug Use. Journal Religion of Health, 53(1), 37-45.

- Brewer, G., Robinson, S. et al. (2014). The Influence of Religious Coping and Religious Social Support on Health Behaviour, Health Status and Health Attitudes in a British Christian Sample. Journal Religion of Health, 54, 2225-2234.

- Bruce, M.A., Beech, B.M. et al. (2016). Spirituality, Religiosity, and Weight Management Among African American Adolescent Males: The Jackson Heart KIDS Pilot Study. Journal of Behavioral Medicine, 42(3), 183-189.

- Caravaca, J.A., Jules, M. et al. (2015). The role of family relations, spirituality and entertainment in moderating peer influence and drug use among students of eight universities from five countries in Latin America and three from the Caribbean. Texto $\mathcal{E}$ contexto enfermagen, 24, 106-116.

- Carrasco, Y. (2015). La religión y su influencia en las conductas de salud (Tesis doctoral). Facultad de Ciencias de la Educación, Universidad de Huelva, Huelva. Recuperado de http://rabida.uhu.es/dspace/bitstream/handle/1027 2/11985/La_religion_y_su_influencia.pdf?sequence $\underline{=2}$

- Castellanos, F., Lucero, A. (2012). Prácticas religiosas en un grupo de personas mayores en situación de discapacidad y pobreza. Investigación en Enfermería: Imagen y Desarrollo, 14(2), 51-61.

- Da Rocha, N., Fernandes, L. \& Mendes, A.M. (2015). Association between alcohol and tobacco consumption and religiosity. Acta Paulista de Enfermagem, 28(6), 546-552.

- Da Trinidade, E.M., Ángel, L. \& Chad, L.E. (2013). La Interculturalidad en el mundo globalizado y sus impactos en el proceso salud-enfermedad: contribuciones de la enfermería. Index de Enfermería, 22(4), 214-218.

- Debnam, K., Holt, C.L. \& Clark, E.M. (2012). Relationship between religious social support and general social support with health behaviors in a national sample of African Americans. Journal of Behavioral Medicine, 35, 179-189.

- De Diego, R. \& Badanta, B. (2017). Health impact of religious practices and beliefs associated with de Church of Jesus Christ of Latter-Day Saints. Journal Religion of Health, 1-10.

- Gabriela, S., Ibanhes, G. et al. (2015). Calidad de vida/espiritualidad, religión y creencias personales de adultos y ancianos renales crónicos en 


\section{Cultura de los Cuidados}

hemodiálisis. Revista Latino-Americana de Enfermagem; 22(6), 911-917 Recuperado de http://www.scielo.br/scielo.php?script=sci arttext \&pid=S0104-11692014000600911.

- García, G., Ellison, C.G. et al. (2013). Religion and Selected Health Behaviors Among Latinos in Texas. Journal Religion of Health, 52, 18-31.

- Glicksman, A. \& Koropeckyj-Cox, T. (2009). Aging among Jewish Americans: Implications for understanding religion, ethnicity, and service needs. Gerontologist, 49(6), 816-827.

- Glicksman, G.G. \& Glicksman, A. (2017). We used to say "Zei gezunt!" (Be well!): Do American Jews still exhibit distinctive health behaviors? J Relig Spiritual Aging, 1-8.

- Hassan, H.S. (2015). Effects of Religious Behavior on Health-Related Lifestyles of Muslims in Malaysia. Journal Religion of Health, 54, 1238-1248.

- Hayward, R.D., Krause, N. \& Pargament, K. (2017). The Prevalence and Antecedents of Religious Beliefs About Health Control in the US Population: Variations by Race and Religious Background. Journal Religion of Health; 1-18.

- Janssen-Niemeijer, A.J., Visse, M., Van Leeuwen, R., Leget, C. \& Cusveller, B.S. (2017) The Role of Spirituality in Lifestyle Changing Among Patients with Chronic Cardiovascular Diseases: A Literature Review of Qualitative Studies. Journal Religion of Health, 1-18.

- Koenig, H.G. (2015). Religion, spirituality, and health: a review and update. Advances in mind-body medicine, 29(3), 19-26.

- Koufakis, T. (2017). Efects of Orthodox religious fasting on human health: a systematic review. European Journal of Nutrition, Dec 56(8), 2439-2455.

- Kretchy, I., Owusu-Daaku, F. \& Danquah, S. (2013). Spiritual and religious beliefs: Do they matter in the medication adherence behaviour of hypertensive patients? BioPsychoSocial Medicine, 7(1), 15.

- Linardakis, M., Papadaki, A., Smpokos, E. et al. (2015). Are religiosity and prayer use related with multiple behavioural risk factors for chronic diseases in European adults aged 50+ years? Public Health, 129(5), 436-443.

- Lopes, E.C., Freire, P.C. et al. (2014). Quality of life, depressive symptoms and religiosity in elderly adults: A cross-sectional study. Texto contexto enfermagem, 23(3), 648-655.

- Menéndez, E. (1994) Hacia una práctica médica alternativa, hegemónica y autoatención (gestión) en salud. México: CIESAS.

- Moreno, L. (2008). Reflexiones sobre el trayecto salud-padecimiento-enfermedad-atención: una mirada socioantropológica. Salud Pública de México, 49(1), 63-70.

- Navas C \& Villegas H. (2006) Espiritualidad y salud. Rev. Cienc. Salud 1 (27): 29-45. Recuperado de http://servicio.bc.uc.edu.ve/cdch/saberes/a1n1/art5 .pdf.

- Orozco-Parra, C. L. \& Domínguez-Espinosa, A. (2014). Diseño y validación de la Escala de Actitud Religiosa. Revista de Psicología, 23(1), 3-11. DOI: 10.5354/0719-0581.2014.32868.

- Padela A.I., Vu, M. et al. (2016). Religious beliefs and mammography intention: findings from a qualitative study of a diverse group of American Muslim women. Psycho-Oncology, 25, 1175-1182.

- Pitel, L., Madarasova, A. et al. (2012). Gender differences in the relationship between religiosity and health-related behaviour among adolescents. J Epidemiol Community Health, 66, 1122-1128.

- Planet, A.I. (2012). Diversidad religiosa y migración en la España de hoy: análisis y actitudes del marco legal. Anuario de Inmigración en España, 104-124.

- Wang, Z., Koenig, H.G. \& Al Shohaib, S. (2015). Religious involvement and tobacco use in mainland China: A preliminary study. BMC Public Health, 15(1), 155-164.

- Watkins, Y.J., Quinh, L.T. et al. (2013). Spiritual and Religious Beliefs and Practices and Social Support's Relationship to Diabetes Self-Care Activities in African Americans. Diabetes Educ, 29(2), 231-239.

- Zini, A., Sgan-Cohen, H.D. \& Marcenes, W. (2012) Religiosity, Spirituality, Social Support, Health Behaviour and Dental Caries among 35- to 44-YearOld Jerusalem Adults: A Proposed Conceptual Model. Caries Research, 46, 368-375. 